\title{
Esporotricose zoonótica na região sul do Rio Grande do Sul (Brasil) e revisão da literatura brasileira
}

\section{Zoonotic sporotrichosis in Southern region of Rio Grande do Sul State (Brazil) and review Brazilian literature}

\author{
Márcia de Oliveira Nobre, ${ }^{*}$ Mario Carlos A. Meireles, ${ }^{\star}$ Dilma Torres Caetano, ${ }^{\star \star}$ Flávio Faé, ${ }^{\star \star}$ \\ João Manoel Chapoon Cordeiro, ${ }^{\star}$ Rostanda Marti Meireles, ${ }^{\star \star \star}$ Carin E. Appelt, ${ }^{\star \star \star \star}$ Laerte Ferreiro ${ }^{\star \star \star \star}$
}

\begin{abstract}
Resumo
Este trabalho relata um caso de esporotricose felina com acometimento de três pessoas e ainda um caso de esporotricose humana a partir da arranhadura de um gato saudável, na região sul do estado do Rio Grande do Sul (Brasil). O gato apresentava uma dermatite ulcerativa generalizada, principalmente na cabeça e pescoço e evoluiu para morte. Durante internação em Clinica Veterinária, arranhou e/ou mordeu o médico-veterinário e dois atendentes, que posteriormente apresentaram lesões ulcerativas nas mãos e antebraços, sendo dois casos com comprometimento linfático. $O$ paciente do quarto caso de esporotricose humana também apresentava lesões no antebraço, que surgiram após ter sido arranhado por um gato saudável. O S. schenckii foi isolado a partir das lesões dos quatro pacientes humanos, que posteriormente foram tratados exitosamente com iodeto de potássio ou itraconazol. Os casos de esporotricose felina, canina e zoonótica relatados no Brasil são apresentados.
\end{abstract}

Palavras-chave: Sporothrix schenckir, esporotricose; gato; zoonose.

\begin{abstract}
This work report cases of sporotrichosis feline associated with three human outbreaks sporotrichosis and still a case human starting from the scratch of a healthy cat, in the south area of the state of Rio Grande do Sul (Brazil). The cat presented dermatitis widespread ulcerative, mainly in the head and neck and progressed at died. During internment in Veterinary Clinic scratched with lymphatic comproctor and two attendants, that later presented lesions ulcerative in the hands and forearm, in two cases appere patient of the fourth case human sporothrichosis also presented lesions in the forearm appeared after scratched by a healthy cat. $S$. schenckii was isolated from the four patients lesions, which that later were treated are presented.
\end{abstract}

Keywords. Sporothrix schenckii; sporotrichosis; cat; zoonosis.

\section{Introdução}

A esporotricose é uma micose subcutânea, causada pelo fungo dimónfico Sporothrix schenckii, que acomete o homem e uma grande variedade de animais como: gatos, cães, cavalos, bois, camelos, golfinhos, mulas, porcos, ratos entre outros. Embora tenha distribuição mundial, a esporotricose ocorre com maior freqüência em regiões de clima tropical. A forma sapróbia, filamentosa, do $S$. schenckii na natureza é encontrada em solos ricos em matéria orgânica e na casca dos caules de arbustos e árvores em regiōes de climas quentes e úmidos, ou in vitro quando cultivado a $25^{\circ} \mathrm{C}$, enquanto a forma leveduriforme representa a fase de parasitismo, sendo encontrada em tecidos infectados ou em cultivos a $37^{\circ} \mathrm{C}$ (Lacaz et al., 1991; Kwon-Chung e Bennett, 1992).

A esporotricose ocorre a partir de ferimentos perfurantes $e$ exposição ao organismo infectante existente no meio ambiente. A esporotricose cutânea se apresenta nas seguintes formas: fixa, disseminada e linfática e raramente evolui como

\footnotetext{
1. Corresponding author. Mailing address: Faculdade de Veterinária, Universidade Federal de Pelotas, Pelotas, RS, Brasil, Campus Universitário, Cep

* Faculdade de Veterinária, Universidade Federal de Pelotas - UFPEL.

** Curso de Medicina, Fundação Universidade do Rio Grande - FURG.

**Faculdade de Medicina, Universidade Federal de Pelotas - UFPEL.

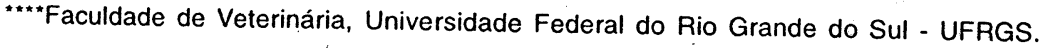


micose sistêmica. Os principais sintomas clínicos e lesões são nódulos, úlceras, crostas e enfartamento ganglionar seguindo a cadeia linfática (Kwon-Chung e Bennett, 1992; Arenas, 1993; Donadel et al., 1993 Farias et al., 1997).

Segundo a revisão realizada por Ferreiro (1984), os primeiros casos da esporotricose documentados em carnívoros domésticos no Brasil foram relatados em gatos (Freitas et al., 1956) e em cães (Souza, 1957) e, no estado do Rio Grande do Sul (RS), o primeiro caso foi reportado em dois cães (Londero et al., 1964). Almeida et al. (1955) citaram, pela primeira vez no Brasil, a possibilidade do ferimento por arranhadura de gato ter sido porta de entrada do $S$. schenckii, levando ao desenvolvimento de esporotricose humana, muito embora o animal não tenha sido examinado. A esporotricose, em animais, no Brasil tem sido considerada rara (Migliano et al., 1963; Larsson et al., 1989; Marques et al., 1993). Segundo Larsson (2000), até 1997, no estado de São Paulo, o maior número de casos de esporotricose foi relatado por Freitas et al. (1965), com oito casos em felinos e 12 em cães. Nos últimos anos vários casos de esporotricose felina foram descritos na região sudeste do Brasil, com maior ocorrência no Rio de Janeiro (Baroni et al., 1998; Andrade et al., 1999; Brustein et al., 1999; Brustein et al., 2000; Campos et al., 2000; Costa et al., 2000; Schiavo et al., 2000; Schubach et al., 2000a; Schubach et al., 2000b; Souza et al., 2000; Liparasi et al., 2001; Schubach et al., 2001a; Schubach et al., 2001b), seguido por São Paulo (Marques et al., 1993; Farias et al, 1995; Marques et al., 1997a; Marques et al., 1998; Larsson, 2000; Fleury et al., 2001) e Minas Gerais (Nogueira et al., 1995). Proporcionalmente ao aumento de relato de casos de esporotricose felina na região sudeste do Brasil, também tem aumentado o número de relatos desta micose em humanos (Larsson, 1989; Marquies et al., 1993; Farias et al., 1995; Nogueira et al., 1995; Marques et al., 1997; Marques et al., 1998; Andrade et al., 1999; Schubach, et al., 2000a; Schubach, et al., 2000b; Souza et al., 2000; Laparisi et al., 2001; Fleury et al., 2001; Schubach et al., 2001b).

Neste trabalho descrevemos casos de esporotricose felina com transmissão para humanos e ainda um outro caso de esporotricose humana adquirido através da arranhadura de felino hígido, ocorridos na região do litoral sul do Rio Grande do Sul.

\section{Material e métodos}

Foi levado para atendimento em março de 1996, em clínica localizada na zona urbana da cidade de Rio Grande (RS-Brasil) um felino doméstico (Felis catus) macho, sem raça definida, com idade aproximada de cinco anos. O exame clínico do felino mostrou temperatura retal de $38,4^{\circ} \mathrm{C}$, mucosas rosadas, deformação do espelho nasal e presença de secreção nasal purulenta bilateral, estertores pulmonares úmidos, sinais de desidratação moderada e lesões cutâneas circulares, elevadas caracterizadas por alopecia, crostas e ulceração central, das quais drenava um exsudato castanhoavermelhado. As manifestações cutâneas eram localizadas principalmente na cabeça e pescoço, atingindo também membros e cauda (Figura1). O felino foi tratado com medicamentos não-específicos e em oito dias morreu. Durante o periodo em que estava hospitalizado infectou, através de arranhadura ou mordedura, o médico-veterinário $e$ dois atendentes.

As lesões nos humanos apareceram entre o $11^{\circ}$ e $14^{\circ}$ dias após a arranhadura ou mordedura e localizaram-se nas mãos

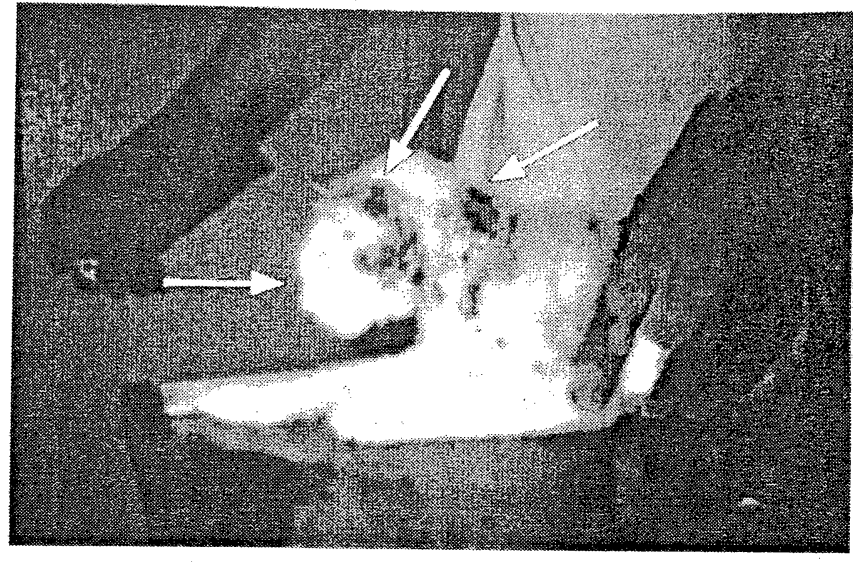

Figura 1: Felino doméstico com esporotricose apresentando lesões ulcerativas na região frontal da cabeça, orelhas e deformação do espelho nasal e com drenagem de exsudato castanho-avermelhado das lesões.

e antebraços, com nódulos que supuraram, ulceraram e drenaram exsudato marrom-avermelhado, formando crostas. Em um caso a manifestação da esporotricose foi na forma cutânea fixa e, em dois casos, evoluiu para a forma linfática, com manifestação de linfangite ascendente (Figura 2). Foi diagnosticado um outro caso humano de esporotricose, sem relação com os anteriores, sendo que a lesão surgiu após 20 dias da arranhadura de um gato aparentemente saudável. A lesão inicial estava localizada no antebraço e evoluiu para linfangite ascendente com formação de nove nódulos, que ulceraram. Após confirmação do diagnóstico micológico, os pacientes foram tratados com iodeto de potássio (1g três vezes ao dia) ou itraconazol (100mg uma vez ao dia).

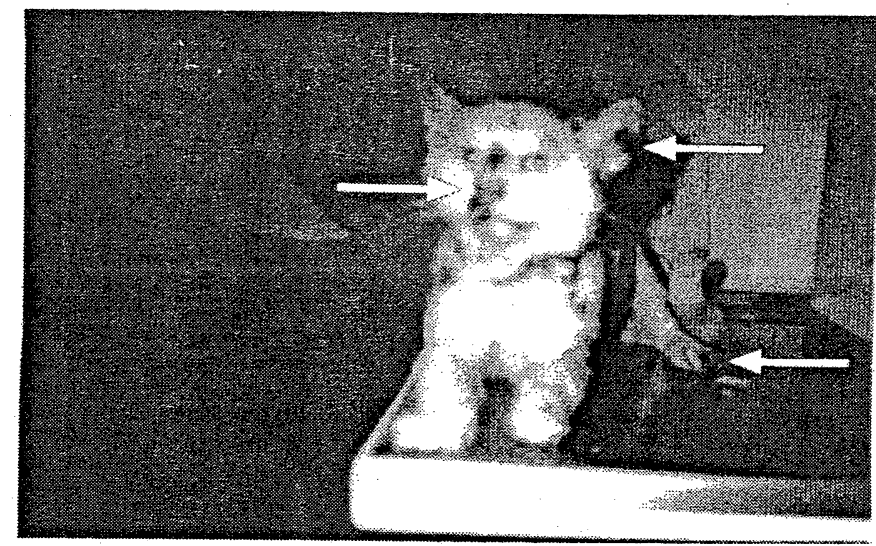

Figura 2: Lesōes de esporotricose nos antebraços dos pacientes humanos: a) forma cutânea com linfangite ascendente; b) forma cutânea fixa com a formação de uma única úlcera.

Foram colhidas secreções das lesões ulceradas, as quais foram semeadas em Mycobiotic agar' e incubadas a $25^{\circ} \mathrm{C}$ por 20 dias e posteriormente foi obtida a reversão para a Bacto mycobiotic Agar, Laboratórios Dfico Ltda, Detroit, Michigan,
USA. 
forma leveduriforme cultivando em ágar sangue (8\% de sangue ovino desfibrinado) e incubando a $37^{\circ} \mathrm{C}$ durante três dias, sendo todos os cultivos caracterizadas macro e micromorfologicamente. A partir do isolamento da forma filamentosa do $S$. schenckii, foi realizada inoculação em testículo de rato (Rattus novergicus-cepa wistar/UFPEL), para recuperação do agente e exame histopatológico utilizando coloração PAS (PeriodicSchiff Acid).

\section{Resultados e discussão}

As lesões no felino, como nos humanos, foram similares àquelas descritas na literatura (Barsanti, 1984; Diaz, 1987; Larsson et al., 1989; Kelly e Clark, 1991; Donadel et al., 1993; Accioly et al.,1993; Marques et al., 1993; Smilack, 1993; Nogueira, 1995; Baroni et al., 1998; Marques et al., 1998; Schubach e schubach, 2000; Schubach et al., 2000b), sendo as formas clínicas: cutânea fixa e a linfática, as quais foram apresentadas pelos pacientes estudados, as mais comuns em humanos (Diaz, 1987; Marques et al., 1997b; Schubach et al., 2001b), principalmente localizadas nos membros superiores (Donadel et al., 1993; Marques et al., 1997b; Schubach et al., 2001b). A contaminação e desenvolvimento da esporotricose em veterinários, estudantes de veterinária e atendentes tem sido descrito na literatura (Zamri-saad et al., 1990; Kelly e Clarck, 1991; Marques et al., 1993; Smilack, 1993; Nogueira et al., 1995; Reed et al., 1995; Fleury et al., 2001; Liparisi et al., 2001), demonstrando o risco ocupacional na cadeia epidemiológica desta micose. As lesões no felino localizavam-se na cabeça, pescoço, membros e cauda, caracterizando a forma cutânea disseminada que comumente acomete os felinos (Barsanti, 1984; Farias et al.,1995; Nogueira et al., 1995; Marques et al., 1998; Andrade et al., 1999; Brustein et al., 1999; Schiavo et al., 2000; Schubach e Schubach, 2000; Schubach et al., 2000b; Schubach et al., 2001c). Neste estudo não foram realizados testes para diagnóstico de retroviroses, causadas pelos virus da leucemia felina (FeLV) e vírus da imunodeficiência felina (FIV), embora nos últimos anos, tenham sido relatados casos de esporotricose felina em gatos FeLV e lou FIV positivos (comunicação pessoal Ferreiro, 2001; Schubach et al., 2001c; Souza, 2001), o que justificaria o quadro clínico de maior gravidade desta micose e o maior número de casos em felinos nas últimas décadas.

Os cultivos incubados a $25^{\circ} \mathrm{C}$ apresentaram inicialmente colônias brancas, tornando-se escuras com o passar do tempo; na microscopia foi observada a presença de hifas finas septadas, com abundantes conídios sésseis ao longo da hifa e raros conídios arranjados na extremidade do condióforo, em forma de "margarida" (Lacaz et al., 1991; Kwon-Chung e Bennett, 1992). Os cultivos incubados a $37^{\circ} \mathrm{C}$ apresentaram colônias de cor creme e consistência cremosa. $O$ estudo micromorfológico demonstrou células leveduriformes, ovaladas, com formato de "charuto", característica do S. schenckii, forma esta, também observada no exame histopatológico do testículo do rato, que desenvolveu orquite bilateral em 15 dias (Lacaz et al., 1991; Kwon-Chung e Bennett, 1992).

O uso do tratamento para esporotricose humana com iodeto de potássio ou itraconazol resultou em desaparecimento das lesões (Dunstan et al., 1986; Restrepo et al., 1986; Accioly et al., 1993; Pena et al., 1999) com, no máximo, 130 dias de medicação, não havendo formação de novos nódulos. O felino não foi tratado especificamente para a micose e, devido ao precário estado geral, evoluiu para a morte. Ressalta-se que, na maioria dos casos de esporotricose, os felinos infectam outros animais ou pessoas (Kenis et al., 1994; Brustein et al., 1999) e morrem naturalmente ou sofrem eutanásia (Farias et al., 1995; Marques et al., 1997a; Marques et al., 1998; Schubach et al., 2000b), freqüentemente devido à dificuldade no diagnóstico precoce da micose. Schubach et al. (2001c) salientam que o maior obstáculo para o tratamento eficaz de felinos acometidos de esporotricose é a falta de adesão do proprietário ao esquema terapêutico proposto. Os felinos são bastante sensiveis ao tratamento com iodeto de potássio, podendo desenvolver iodismo (Farias et al., 1997; Costa et al., 2000; Larsson, 2000), embora o tratamento tenha êxito em alguns casos (Dunstan et al., 1986; Gonzales et al., 1989;). Estudos recentes indicam o uso dos azóis no tratamento da esporotricose felina (Schubach e Schubach, 2000; Schubach et al., 2001c) havendo relatos do uso eficiente do cetoconazol (Nakamura et al., 1996; Schiavo et al., 2000) e do itraconazol (Costa et al., 2000) este último baseado em sete anos de observações clínicas (Larsson et al., 2000). Estes antifúngicos, cetoconazol e itraconazol, foram testados in vitro demonstrando atividade contra cepas de S. schenckii (Casali, 1995). Também tem sido utilizada a terbinafina no tratamento da esporotricose felina (Schubach et al., 2001c).

Os nossos resultados demonstram, a exemplo de trabalhos realizados em outras regiões do Brasil e no exterior, a importância epidemiológica do felino doméstico na esporotricose humana (Dunstan et al., 1986; Larsson et al., 1989; Kelly e Clarck, 1991; Marques et al., 1993; Reed et al., 1993; Smilack, 1993; Marques et al., 1997a; Marques et al., 1997b; Andrade et al., 1999; Schubach et al., 2000b; Schubach et al., 2000a; Fleury et al., 2001; Schubach et al., 2001b). Epidemiologicamente, são importantes fontes de infecção os felinos machos não castrados e de livre acesso à rua, pelos hábitos de esconderem-se entre plantas, rebolcarem-se no solo, marcarem territórios com as unhas em cascas de árvores e arranharem-se entre si em lutas ou em brincadeiras (Larsson et al., 1989; Farias et al., 1997; Schubach et al., 2000b). Estudos relatam o isolamento de $S$. schenckii de unhas (Schubach et al., 2000b; Schubach et al., 2001a; Souza et al., 2001) e cavidade bucal (Schubach et al., 2001a) de gatos acometidos com esporotricose destacando o risco de inoculações do agente através da arranhadura e mordedura.

$\mathrm{Na}$ região sudeste, particularmente no Rio de Janeiro, zona litorânea de clima quente e úmido, o relato de casos de esporotricose felina a partir de 1998 tem aumentado (Tabela 1), seja como casos isolados ou atingindo grupos de animais até estudos epidemiológicos, o que levou ao registro de centenas de animais comprovadamente com esporotricose (Baroni et al., 1998; Andrade et al., 1999; Brustein et al., 1999; Brustein et al., 2000; Costa et al., 2000; Schiavo et al., 2000; Schubach et al., 2000a; Schubach et al., 2000b; Souza et al., 2000; Campos et al., 2001; Liparasi et al., 2001; Schubach et al., 2001a; Schubach et al., 2001b). Em São Paulo, a partir de 1989 foram relatados 43 casos de esporotricose felina (Larsson et al., 1989; Marques et al., 1993; Farias et al., 1995; Marques et al., 1997a; Marques et al., 1998; Larsson et al., 2000; Fleury et al., 2001) e em Minas Gerais um (Nogueira et al., 1995). Estudo desenvolvido no Rio Grande do Sul demonstrou a ausência da esporotricose ou a falta de diagnóstico des- 
ta micose em felinos residentes na região de Porto Alegre e municípios vizinhos (Oliveira et al., 1999). Entretanto, recentemente foram diagnosticados dois casos de esporotricose felina em Porto Alegre (Comunicação pessoal - Ferreiro, 2001), e outros 40 casos, na cidade de Rio Grande (Castro et al., 2001; Souza, 2001).

Os casos zoonóticos envolvendo felinos e humanos aumentaram (Larsson et al., 1989; Marques et al., 1993; Farias et al.,

Tabela 1: Casos de esporotricose felina, canina e zoonótica no Brasil (1956-2001).

\begin{tabular}{|c|c|c|c|c|c|}
\hline \multirow[t]{2}{*}{ AUTOR E DATA } & \multirow[t]{2}{*}{ PERÍODO } & \multirow[t]{2}{*}{ ESTADO } & \multicolumn{3}{|c|}{ CASOS DE ESPOROTRICOSE } \\
\hline & & & Felinos & Caninos & Humanos \\
\hline Freitas et al., 1956 & - & SP & 1 & $\ldots$ & $\ldots$ \\
\hline Souza, 1957 & - & SC & $\ldots$ & 1 & $\ldots$. \\
\hline Migliano et al., 1963 & - & SP & $\ldots$ & 2 & $\ldots$ \\
\hline Londeiro et al., 1964 & - & RS & $\ldots$ & 2 & $\ldots$ \\
\hline Freitas et al., 1965 & $1963-1964$ & SP & 8 & 12 & \\
\hline Larsson et al., 1989 & - & SP & 1 & $\ldots$ & 3 \\
\hline Marques et al.,1993 & - & $\mathrm{SP}$ & 5. & .. & 3 \\
\hline Farias et al., 1995 & - & SP & 1 & $\ldots$ & 1 \\
\hline Nogueira et al., 1995 & - & MG & 1 & $\ldots$ & 2 \\
\hline Marques et al., 1997a & - & $\mathrm{SP}$ & 1 & $\ldots$ & 2 \\
\hline Marques et al., 1998 & - & SP & 1 & $\ldots$ & 2 \\
\hline Baroni et al., 1998 & - & RJ & 1 & $\ldots$ & $\ldots$ \\
\hline Andrade et al., 1999 & $=$ & RJ & 2 & $\ldots$ & 2 \\
\hline Brustein et al., 1999 & 1997-1999 & RJ & 12 & 1 & $\ldots$ \\
\hline Oliveira et al., 1999 & $-\cdots$ & RS & $\cdots$ & $\cdots$ & $\ldots$ \\
\hline Brustein et al., 2000 & $1997-2000$ & RJ & 45 & $\cdots$ & $\ldots$ \\
\hline Costa et al., 2000 & - & RJ & 4 & $\cdots$ & $\ldots$ \\
\hline Larsson et al., 2000 : & $1986-1987$ & SP & 30 & $\ldots$ & $\cdots$ \\
\hline Schiavo et al., 2000 & - & RJ & 1 & $\ldots$ & $\ldots$ \\
\hline Schubach et al., $2000 a$ & - & RJ & 73 & 3 & 21 \\
\hline Schubach et al., $2000 \mathrm{~b}$ & $-1998-2000$ & RJ & 3 & $\ldots$ & 3 \\
\hline Souza et al., 2000 & $\therefore$ & RJ & 11 & $\ldots$ & 1 \\
\hline Campos et al., 2001 & $1998-2000$ & RJ & 2 & $\ldots$ & $\ldots$ \\
\hline Castro et al., 2001 & $1997-2001$ & RS & 22 & $\cdots$ & 1 \\
\hline Ferreiro, 2001 & & RS & 2 & $\ldots$ & $\ldots$ \\
\hline Fleury et al., 2001 & $1998-1999$ & SP & 4 & $\cdots$ & 4 \\
\hline Liparisi et al., 2001 & - & RJ & 1 & $\cdots$ & 2 \\
\hline Schubach et al. $2001 \mathrm{a}$ & $1998-2000$ & RJ & 119 & $\cdots$ & $\ldots$ \\
\hline Schubach et al. 2001b & $1998-2000$ & RJ & 148 & $\ldots$ & 81 \\
\hline Schubach et al., 2001d & - & RJ & $\ldots$ & 4 & $\cdots$ \\
\hline Souza, 2001 & 1999-2001 & RS & 18 & $\ldots$ & \\
\hline
\end{tabular}

1995; Nogueira et al., 1995; Marques et al., 1997a; Marques et al., 1998; Castro et al., 2001; Fleury et al., 2001), principalmente no Rio de Janeiro com 110 casos documentados (Andrade et al., 1999; Schubach et al., 2000a; Schubach et al., 2000b; Souza et al., 2000; Liparasi et al., 2001; Schubach et al., 2001b) (Tabela 1), enquanto ocorreu um decréscimo nos relatos de casos de esporotricose canina no Brasil nos últimos anos (Brustein et al., 1999; Schubach et al., 2000a; Schubach et al., 2001d) (Tabela 1), possivelmente pela micose ser menos grave em cães e também por ser facilmente confundida com outras doenças (Schubach et al., 2001d).

Na cidade de Rio Grande (RS/ Brasil), de onde são procedentes os casos aqui relatados e outros casos foram descritos, neste último ano (Castro et al., 2001; Souza, 2001), o clima é frio, diferindo das condições climáticas do Rio de Janeiro, tendo em comum a condição de litoral, com alta umidade, podendo ser estes os fatores que facilitem a condição ambiental para a manutenção do $S$. schenckii no meio ambiente. Estudos futuros em relação ao ambiente de desenvolvimento do $S$. schenckii serão necessários, a fim de esclarecer a maior freqüência da doença em determinados nichos.

Este relato demonstra o crescimento do número de casos de esporotricose na região do litoral sul do Rio Grande do Sul, enfatizando o risco para humanos das arranhaduras e mordeduras de gatos hígidos ou com esporotricose, e salienta o risco potencial de transmissão desta micose, que pode ser considerada como emergente na região de abrangência deste estudo, para o médico-veterinário de pequenos animais e seus atendentes.

\section{Referências}

ACCIOLY FILHO, A. J. W.; JACQUES, C. S. C.; LEVERONE, A.P.; OLIVEIRA, J. C.; MENDONÇA, I. R. S. M.; AZZULAY, R. D. Itraconazol no tratamento da esporotricose. Relato de três casos. An. Bras. Dermatol., v. 68, p. 117-119, 1993.

ALMEIDA, F.; SAMPAIO, S. A. P.; LACAZ, C. S.; FERNANDES, J. C. Dados estatísticos sobre a esporotricose - análise de 344 casos. Anais Bras. Derm. e Sif., v.1, p. 9-12, 1955.
ANDRADE, B. P. J.; BRUSTEIN, R.; RODRIGUES, M. R. Esporotricose: quando gatos e proprietários são infectados. CONGRESSO INTERNACIONAL DE MEDICINA FELINA, 1., 1999, Rio de Janeiro. Anais..., 1999. p. 26.

ARENAS, R. Micologia Médica ilustrada, 1. ed. México: Nueva Editorial Interamericana, 1993.

BARONI, F. A.; CAMPOS, S. G.; DIREITO, S. M. Esporotricose em gato (descrição de um caso). R. Bras. Med. Vet., v. 20, p. 25-27, 1998. 
BARSANTI, J: A. Sporotrichosis. In: GREENE, C. E. (Ed.). Clinical microbiology and infectious diseases of the dog and cat. Saunders, Philadelphia, 1984, p. 722-727.

BRUSTEIN, R.; DAHER, G. A.; OLIVEIRA, J. G. D.; ANDRADE, B.P.J.; SILVA, C. P.; SILVA, C. B. G.; LUCIANO, S. A. J.; FERNANDES, V. N. Esporotricose em felinos domésticos - Ocorrência em pacientes do Serviço de Diagnósticos Clínicos/Serviço de Patologia Animal do Instituto Municipal de Medicina Veterinária "Jorge Vaitsman" (IJV). CONGRESSOINTERNACIONAL DE MEDICINA FELINA, 1., 1999, Rio de Janeiro: Anais..., 1999. p. 25.

ANDRADE, B. P. J.; ALMEIDA, T. M.; SILVA, K. P.; DAHER, G. A.; CARNEIRO, C. O.; FERREIRA, B. G. Esporotricose felina na cidade do Rio de Janeiro e alguns municípios vizinhos. Rev. Bras. Ciência Vet., v. 7-suplemento, p. 132, 2000.

CAMPOS, S. G.; PEREIRA, M.P.D.; MARTINS, J.M. P. .M.; RAMADINHA, R.H.R.; FLAUSINO, G.; SCHIAVO, P. Prevalência de fungos em felinos com lesões características de micoses examinados de 1998 a 2000 no Instituto de Veterinária da UFRRJ. CONGRESSO INTERNACIONAL DE MEDICINA FELINA, 2., 2001, Rio de Janeiro. Anais..., 2001. p. 28.

CASALI, A. Composição lipídica e susceptibilidade a drogas antifúngicas de diferentes amostras de Sporothrix schenckii. 1995. 167 f. Dissertação (Mestrado) - Instituto de Ciências Biológicas Universidade Federal de Minas Gerais, 1995.

CASTRO, A. P.; NOBRE, M. O.; CAETANO, D.; SOUZA, L. L.; MEIRELES, M. C. A.; FERREIRO, L. Recurrência de esporotricose em gatos com envolvimento zoonótico. Ciência Anim., v. 11, p. 187, 2001.

COSTA, D. M.; MOREIRA, N. S.; TOLEDO-PIZA, E.; RIBEIRO, L. A. F. Esporotricose felina: relato de quatro casos no Rio de Janeiro. Rev. Bras. Ciência Vet., v. 7-suplemento, p. 131, 2000.

DIAZ, I. C. Esporotricosis. Rev. Med. Uruguay, v. 3, p. 135-147, 1987. DONADEL, K. W.; REINOSO, Y. D.; OLIVEIRA, J. C.; AZULAY, R. D. Esporotricose: revisão. An. Bras. Dermatol., v. 68, p. 45-52, 1993. DUNSTAN, R. W.; LANGHAM, R. F.; REIMANN. K. A.; WAKENELL, P.S. Feline sporothrichosisi: a report of five cases with transmission to humans. J. Amer. Acad. Dermatol. v. 15, p. 37-45, 1986.

FARIAS, M. R.; COSTA, P. R. S.; FRANCO, S. R. V. S.; ROCHA, N. S MARQUES, S. Esporotricose em gato - Relato de caso. CONGRESSO BRASILEIRO DE CLINICOS VETERINÁRIOS DE PEQUENOS ANIMAIS, 17. 1995. Vitória. Anais..., 1995. p. 98.

;FERREIRA, H. Esporotricose canina e felina. Cães \& Gatos, v. 66, p. 30-38, 1997.

FERREIRO, L. Bibliografia sobre micoses, micotoxicoses e micologia experimental em animais no Brasil. Arq. Fac. Vet. UFRGS; v. 12, p. 139-160, 1984

FLEURY, R. N.; TABORDA, P.R.; GRUPTA, A. K.; FUJITA, M. S.; ROSA P. S.; WECKWERTH, A. C.; NEGRÄO, M. S.; BASTAZINI, I. ZOOnotic sporotrichosis. Transmission to humans by infected domestic cat stratching: report of four cases in São Paulo, Brazil. Int. J. dermatol., v. 40, p. 318-322, 2001

FREITAS, D. C.; MIGLIANO, M. F.; ZANI NETO, L. Esporotricose: observação de caso espontâneo em gato doméstico (F. catus, L.) Rev. Med. Vet., v. 5, p. 601-604, 1956.

MORENO, G.; SALIBA, A. M. F.; BOTTINO, A J: MOS, E N Esporotricose em cães e gatos. Rev. Fac. Med. USP, v. 7, p. 381-387, 1965. GONZALEZ, C. J. F;; HERAS, G. M.; LATRE, C. M. V.; GARCIA, J. C. J. A. Feline sporotrichosis: a case report. Mycopathol., v. 108, p. 149154, 1989

KELLY, S. E.; CLARK, W.T. Feline sporotrichosis: a case report with zoonotic involvement. Australian Vet. Pract., v. 21, p. 139-140, 1991. KENNIS R. A.; ROSSER, E. J.; DUNSTAN, R. W. Difficult dermatologic diagnosis (Sporotrichosis in a cat). J.A.V.M.A., v. 204, p. 51-52, 1994. KWON-CHUNG, K. J.; BENNETT, J. E. Sporotrichosis. In: Medical Mycology. Philadelphia: Lea \& Fibeger, 1992, p. 707-729.
LACAZ, C. S.; PORTO, E.; MARTINS, J. E. C. M. Esporotricose e outras micoses gomosas. In: LACAZ, C.S., PORTO, E., MARTINS, J. E. C. M: (Ed.). Micologia médica. 8. ed. São Paulo: Sarvier, 1991, p. 233-247. LARSSON, C.E. Esporotricose. SIMPOSIOBRASILEIROSOBREMICOSES ANIMAIS, 1., 2000, Porto Alegre. Resumos... 2000, p. 66-71.

GONÇALVES, M. A.; ARAUJO, V. C.; DAGLI, M. L. Z.; NETO, C. F. Esporotricosis felina: aspectos clínicos e zoonóticos (1). Rev. Inst. Med. Trop., São Paulo, v. 31, p. 351-358, 1989.

LIPARISI, F.; TORTELLY, R.; CARVALHO, E. C. Q.; MANDARINO, M.; CALIXTO, R.; FLORIDO, P. S. Importância zoonótica da esporotricose felina. CONGRESSOINTERNACIONAL DEMEDICINA FELINA, 2., 2001, Rio de Janeiro. Anais..., p. 25.

LONDERO, A. T.; CASTRO, R. M.; FISCHMAN, O. Two cases of sporotrichosis in dog in Brazil. Sabouraudia 1964; v. 18, p. 273-274. MARQUES, S. A.; FRANCO, S. R. V. S.; CAMARGO, R. M. P.; DIAS, L. D. F.; HADDAD J. R.; FABRIS, V. E. Sporotrichosis in the domestic cat (Felis catus): human transmission. Rev. Inst. Med. Trop., v. 35, p. $327-$ 330, 1993.

;CAMARGO, R. M. P.; HADDAD JUNIOR, V.; FRANCO, S.E. V. S.; MARQUES, M. E. A. Feline sporotrichosis: Human transmission. CONGRESS OF THE INTERNATIONAL SOCIETY FOR HUMAN AND ANIMAL MYCOLOGY. 14., 1997, Parma. Anais..., 1997. p. 109.(a)

;FAGUNDES, L. K.; SUETO, M.; LASTÓRIA, J.C.; DILLON, N. L. Sporotrichosis: survey and clinical aspects from Botucatu school of Medicine, State of São Paulo-Brazil. An. Bras. Dermatol., v. 72, p. 334447, 1997. (b)

; HADDAD, J. R.; MARQUES, M. E. A.; FRANCO, S. R. V. S $\overrightarrow{\mathrm{ROCHA}}$, N. S. Human sporotrichosis: transmitted by feline. An. Bras. Dermatol., v. 73, p. 559-562, 1998.

MIGLIANO, M. F.; FREITAS, D.C.; MORENO, G. Esporotricose em cães. Rev. Fac. Vet. São Paulo, v. 7, p. 225-233, 1963.

NAKAMURA, Y.; SATO, H.; WATANABE, S.; TAKAHASHI, H.; KOIDE, K.; HASEGAWA, A. Sporothrix schenckii isolated from cat in Japan. Mycoses, v. 39, p. 125-128, 1996.

NOGUEIRA, R. H. G.; GUEDES, R. M. C.; CASSALI, G. D.; GHELLER, V. A.; MOREIRA, Y. K. Relato de esporotricose felina (Sporothrix schenckii) com transmissão para o homem: aspectos clínicos, microbiológicos e anatomopatológicos. Arq. Bras. Med. Vet. Zootec, v. 47, p. 43-51, 1995.

OLIVEIRA, I. A.; APPELT, C. E.; FERREIRO, L. Esporotricose: falta de diagnóstico clínico e laboratorial ou inexistência desta micose na grande Porto Alegre. SALÄO DE INICIAÇÃO CIENTÍFICA DA UFRGS, 11. 1999, Porto Alegre. Anais..., 1999, p. 87.

PENA, C.; GARCIA-SILVA, J.; VARELA, J.; ARDAVAIN, M.; PEREIRO, M. $J R$. Esporotricosis cutánea atípica. Respuesta a itraconazol y cirugía. Acta Otorrinolaring Esp., v. 50, p. 485-489, 1999.

REED, K. D.; MOORE, F. M.; GEIGER, G. E.; STEMPER, M. E. Zoonotic transmission of sporotrichosis: case report and review. Clin. Infect. Diseases, v. 16, p. 384-387, 1993.

RESTREPO, A.; ROBLEDO, J.; GOMEZ, I.; TABARE, A. M.; GUTIERREZ, $R$. Itraconazole therapy in lynphangitic and cutaneos sporotrichosis. Arch. Dermatol., v. 122, p. 413-417, 1986.

SCHIAVO, P.A.; CAMPOS, S. G.; RAMADINHA, R.H. R.; FLAUSINO, G. Resposta terapêutica em um caso de esporotricose em gato. Rev. Bras. Ciência Vet., v. 7 - suplemento, p. 132, 2000.

SCHUBACH, T. M. P.; SCHUBACH, A, O. Esporotricose em gatose cães - revisão. Clin. Vet. v. 29, p. 21-24, 2000.

SCHUBACH, T.; MELO, M.; VALLE, A.; GUITIERREZ-GALHARDO, M.; BARROS, M.; MONTEIRO, P.; REIS, R.; CUZZI-MAYA, T.; BLANCO, T.; LOURENÇO, M.; SILVA, N.; ZANCOPÉ-OLIVEIRA, R.; MARZOCHII, K.; SCHUBACH, A. Clinical evolution of feline and canine sporothrichosis in Rio de Janeiro. CONGRESSO BRASILEIRODE CLINICOS VETERINÁRIOS DE PEQUENOS ANIMAIS, 21., 2000, Rio de Janeiro. Anais..., 2000. p. 38.(a) 
SCHUBACH, T. M. P.; OKAMOTO, T:PELLON, I V : MONTEIRO, D. F.; MELO, M.; REIS, R. S.; FIALHO-MONTEIRO, P. C.; SCHUBACH, A. O gato doméstico - fonte de infecção da esporotricose urbana no Rio de Janeiro. CONGRESSOINTERNACIONAL DE MEDICINAFELINA, 2., 2001, Rio de Janeiro. Anais..., 2001. p. 25.(a)

;VALLE, A. C. F.; GUTIERREZ-GALHARDO, M. C.; MONTEIRO P. C. F.; REIS, R. S.; ZANCOPÉ-OLIVEIRA, R. M.; MARZOCHI, K. B. F. SCHUBACH, A. Isolation of Sporothrix schenckii from the nails of domestic cats (Felis catus). Med. Mycol., v. 39, p. 147-149, 2000. (b)

OKAMOTO, T.; PELON, I. V.; MONTEIRO, D. F.; MELO, M.; REIS R. S.; FIALHO-MONTEIRO, P. C.; BLANCO, T. C. M.; CUZZY-MAIA, T.; $\mathrm{SCHUBACH}, \mathrm{A}$. Clínica e terapêutica da esporotricose em gatos naturalmente infectados. Ciência Anim., v. 11, p. 193, 2001.(c)

;SCHUBACH, A.; SILVA, V. F.; FIALHO-MONTEIRO, P. C.; BAR BOSA-SANTOS, E.; DUARTE, R.; CONFORT, E. M.; MARZOCHI, K.B. F.; MARZOCHI, M. C. A. Avaliação da intradermorreação e sorologia para leishmaniose em cães com esporotricose. Ciência Anim, v. 11, p. 193, 2001.(d)

SCHUBACH, A. O.; SCHUBACH, T.M. P.;BARROS, M. B. L.; GUTIERREZGALHARDO, M.C.; NEVES, E.S.; REIS, R. S.;BRUSTEIN, R.; MARZOCHI, K. B. F.; WANKE, B.; FRANCESCONI-DO-VALLE, A. C. Esporotricose zoonótica - abordagem entre medicina humana e veterinária. Ciência Anim., v. 11, p. 192, 2001.(b)

SMILACK, J. D. Zoonotic transmission of sporotrichosis. Clin. Infect. Diseases, v. 17, p. 1075-1076, 1993.

SOUZA, J. .J. Esporotricose em cães. CONGRESSO BRASILEIRO DE MEDICINA VETERINÁRIA, 7., 1957, Recife. Anais..., 1957. p. 367-371. SOUZA, L. L. Sporothrix schenckii: estudo epidemiológico em população de gatos. 2001. 32 f. Dissertação (Mestrado) - Faculdade de Veterinária - Universidade Federal de Pelotas, 2001.

; NOBRE, M. O.; MEIRELES, M. C. A.; NASCENTE, P. S. Isolamento do fungo Sporothrix schenckii das unhas de gatos. CONGRESSO BRASILEIRO DE MICOLOGIA, 2., 2001, Águas de Lindóia. Anais..., 2001, p. 126.

SOUZA, M.D.M.;PERES, M. R.;BERNARDES, M. A.A.G.;GUIMARÃES, L. O. F.; GAZETTA, G. S.; GITTI, C. B.; ABOUD-DUTRA, A. E. Esporotricose felina e a importância zoonótica - relato de caso no Rio de Janeiro, RJ, Brasil. Rev. Bras. Ciência Vet., v.7 - suplemento, p. 131, 2000.

ZAMRI-SAAD, M.; SALMIYAH, T. S.; JASNI, S.; CHENG, B. Y.; BASRI, K. Feline sporotrichosis: an increasingly important zoonotic disease in Malaysia. Vet. Rec., v. 127, p. 480, 1990 